\title{
Static and dynamic detection of axial surface defects on metallic wires by conical triple laser reflection
}

\author{
P. Siegmann, J.C. Martínez-Antón*, E. Bernabeu \\ Departamento de Optica, Universidad Complutense de Madrid, Facultad de C.C. Fisicas, \\ C. Universitaria, Madrid 28040, Spain
}

Received 8 August 2002; received in revised form 25 July 2003; accepted 28 July 2003

\begin{abstract}
The quality of the surface of metallic wires is relevant for different applications. The reflection of a laser beam on the surface of a metallic cylindrical wire provides an efficient way to inspect the quality of its surface. Our interest is focused in the detection of axially oriented defects, which are the most relevant for the wire drawing process. We present a simple interference-geometrical model to describe the light pattern reflected from a wire with defects. This model adequately accounts for the observed results from an industrial prototype developed for the purpose. It incorporates three-laser beams incident on the wire at equidistant locations in its perimeter, which produce three reflection cones with a CCD. This configuration permits to explore the whole perimeter of the wire. Several results are presented, both in static operation and in production line, in agreement with qualitative and quantitative predictions.
\end{abstract}

(C) 2003 Elsevier Ltd. All rights reserved.

Keywords: Wire surface inspection; Conical reflection

\section{Introduction}

In a wire drawing process it is common the apparition of axial surface defects, like scratches, die-lines, ridges, drawing grooves and roughness-strips, due to the drawing process itself. It is very important to detect this defects in the production process, not

*Corresponding author. Tel.: + 34-91-394-4629; fax: + 34-91-394-4683.

E-mail address: jcmartin@fis.ucm.es (J.C. Martínez-Antón). 
only because the quality of the surface of the wires is relevant for different final applications, but also because the apparition of such defects is an indication of quick deterioration of the drawing dies $[1,2]$. On the other hand, these structures modify the properties of the wires in several applications. Therefore, the surface quality should be controlled previous to its use and, if possible, during the production process, what would reduce time and costs in the wire production.

There has been important efforts to perform a superficial characterization of fine metallic wires (30-600 $\mu \mathrm{m}$ of diameter) employing conventional techniques (atomic force microscopy, scanning electron microscope and confocal microscopy), to make a classification of the different kinds of superficial structures, and also to generate a common nomenclature [1,2]. These techniques provide a great quantity of information of the wire surface, but they have the problem of being very slow and need a previous preparation of the samples. Therefore, they are not suitable to be implemented in production line. We have developed and tested an optical technique and an industrial prototype that allows the superficial characterization of metallic wires in a simple and quick way in situ or in the laboratory. It permits to explore the whole perimeter of the wire by using a triple laser reflection around the wire. The obtained experimental results, both in static mode and in production line, are in agreement with the predictions of the presented model.

In previous works the optical model used for the static detection of defects in metallic wires were too simple [3,4]. In this work our intention is to develop a finer model of interaction which adequately addresses some of the features observed in the experimental results. In an optically smooth or perfect wire, the reflected light is distributed along a reflection cone (Fig. 1). Geometrically, every reflection direction

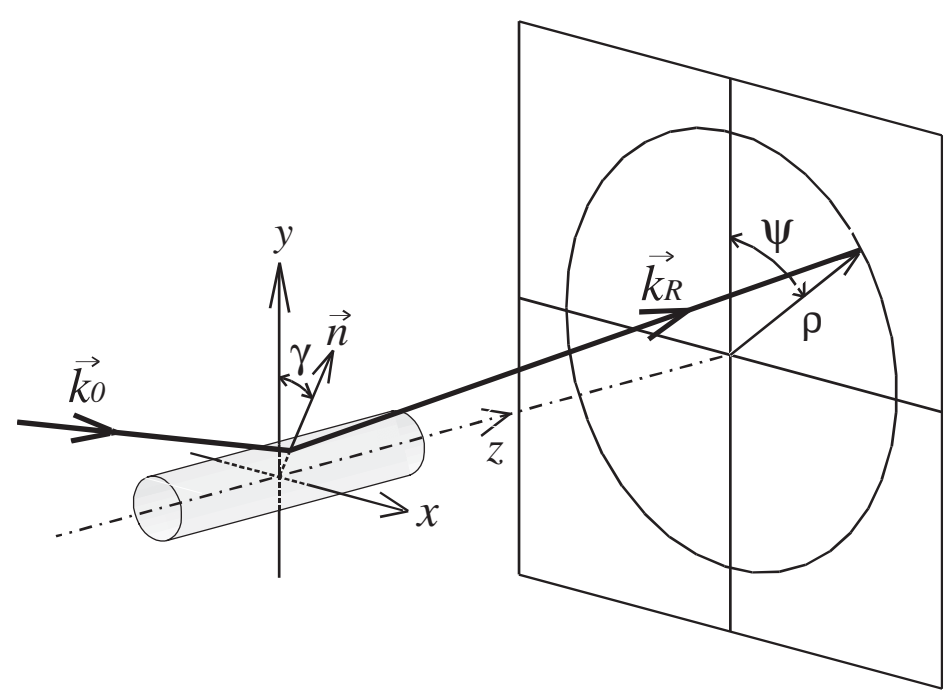

Fig. 1. The oblique incidence of a laser beam on a cylindrical wire lead to a reflection cone geometrically related to the surface of the wire. 
of the cone corresponds uniquely to a point of the wire surface. Therefore, when there is a defect in a particular point of the wire, we encounter a strong loss of intensity or minimum in the corresponding point of the reflection cone. This corresponds more or less to the observed behavior. However, this approximation does not account for the interference effects produced between normal (specular) reflection and the scattering produced at defects. In this contribution, we develop an interferential model more adequate to interpret the observed experimental results.

Any defect with axial structure produces a conical scattered beam which overlaps the reflection cone coming from the smooth wire surface (the basic axial structure). In general terms, the interference of the scattered light with the specular reflection coming from the wire will produce a sort of speckle modulation, as we will see.

Our model deals with smooth surfaces and few isolated axial defects on the wire. However, it establishes the basics to consider the interaction between several possible structures. It is important here to distinguish a textured or homogenous rough surface from a defect or collection of defects. In the first case, it is possible to determine the roughness of the surface by observing the speckle reflected pattern [5]. Our purpose is to analyze the simpler effect of a defect on a smooth surface. We will focus our approach in this last sense but obtain important conclusions to apply in more general cases. From the presented model we will be able to obtain the localization of a defect, and also information about its size parameters (depth and height).

\section{Theoretical model for the reflected light pattern}

In the previous work $[3,4]$ the effect produced by a defect was considered just like a scattering element leading to a gap in the light intensity pattern. Now we basically continue with a geometrical model but incorporating a phase in the reflected wavefronts to take into accounts interferential effects.

In a general measurement configuration we have an oblique incidence on the wire which gives a reflection cone (Fig. 1) containing the surface information of a semicylinder profile. From the symmetry of the problem and the reflection laws, the problem of oblique incidence can be simplified in terms of normal incidence to the wire axis and its projection on a plane normal to the wire. Therefore, we will consider the situation of Fig. 2. It can be demonstrated that, in a general oblique incidence, the projected cone is a version of the normal incidence case [6]. In other words, the $z$ axis propagation components are not relevant for our analysis.

Consider the presence of an isolated continuous defect (sufficiently long in the axial direction) in the wire smooth cylindrical profile (Fig. 2) and let $N_{0}$ equidistant and parallel rays fall on the wire surface (simulating an incident plane wave). All these rays are contained in a plane perpendicular to the wire axis. Let us denote $f(x)$ the wire profile defined by the intersection of that plane with the wire (Fig. 2).

The incident rays may be expressed with the wave vector $\mathbf{k}_{0}=k(0,-1)$, the impact points on the wire surface as $P_{R}=\left(x_{R}, f\left(x_{R}\right)\right.$, the normal vector in these points as $\mathbf{n}_{\mathrm{R}}$, the corresponding reflected rays $\mathbf{k}_{\mathrm{R}}$ and the optical path $l$ from a "reference plane" $\Pi_{0}$ to an "arrival plane" $\Pi_{\psi}$ (defined for an observation angle $\psi$ ). Then, we have for 


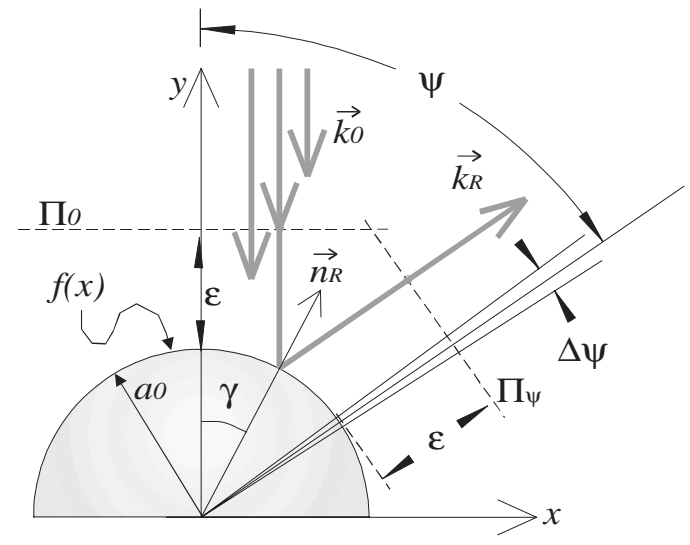

Fig. 2. Collimated rays incident on a small area of a perfect wire surface centered at $\gamma$ are reflected into the angular interval $\psi \pm \Delta \psi / 2$. A more general profile $f(x)$ will scatter into this angular interval from different points of the wire. In fact for a defect located at $\gamma$ we have very few rays reflected at $\psi \pm \Delta \psi / 2$. See text for more details.

each ray

$$
\begin{aligned}
\mathbf{n}_{R} & =\frac{\left(-f^{\prime}, 1\right)}{\sqrt{f^{\prime 2}+1}}, \mathbf{k}_{R}=-2\left(\mathbf{k}_{R} \mathbf{n}_{R}\right) \mathbf{n}_{R}+\mathbf{k}_{0}, \\
l & =\underbrace{\left(a_{0}+\varepsilon\right)-y_{R}}_{\Pi_{0} P_{R}}+\underbrace{\left(a_{0}+\varepsilon\right)-x_{R} \sin \psi-y_{R} \cos \psi}_{\Pi_{\psi} P_{R}},
\end{aligned}
$$

where $f^{\prime}$ is the derivative of $f(x), \varepsilon$ is the separation distance from the planes $\Pi_{0}$ and $\Pi_{\psi}$ to the smooth wire surface, and $\psi$ the observation angle (Fig. 2). In expression $a_{0}+\varepsilon$ can be set to zero without loss of generality.

We define now the detection system in the following way: we count and make interfere in the far field, all the specularly reflected rays whose directions are comprised in an infinitesimal angular interval $\Delta \psi$ (Fig. 2b). The wave amplitude in each angular interval is then (assume the reflectance $R=1$ ):

$$
u(\psi, \Delta \psi)=N_{\Delta \psi} \sum_{n}^{N_{\Delta \psi}} \mathrm{e}^{\mathrm{i} k l_{n}},
$$

where $\psi \pm \Delta \psi / 2$ is the angular interval at which we associate an intensity of $I=u u^{*}$, $N_{\Delta \psi}$ is the number of reflected rays within this angular interval, and $l_{n}$ is the optical path from every ray in this angular interval.

Finally, the intensity profile that we obtain from Eq. (2) will be resampled to simulate the effect of a discrete detector array like a CCD, producing some averaging. Consider a camera with a square pixel with size $p$. Then, the computing angular interval $\Delta \psi$ in should be quite smaller than the angle subtended by the pixel size. The number of incident rays $N_{0}$ is fixed when the condition of a practically constant intensity profile is achieved, i.e. when for $N_{0}+\Delta N_{0}$ we do not get a noticeable change in the normalized intensity profile. 


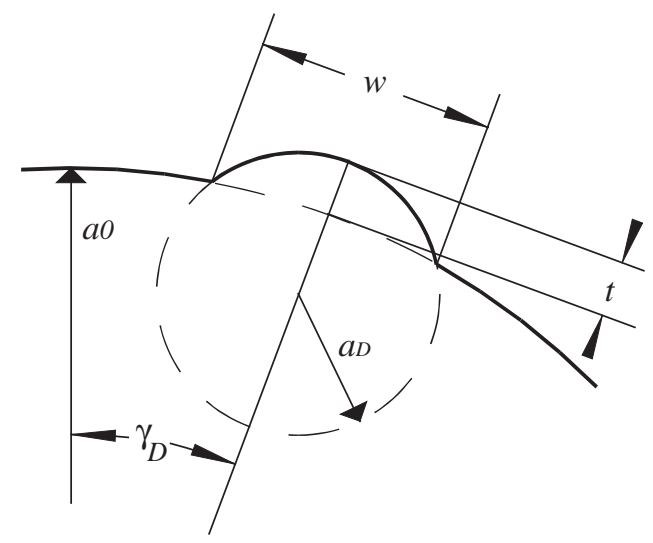

Fig. 3. Semi-cylindrical model for defect description.

For the sake of simplicity we will consider axial extended defects with a cylindrical profile (Fig. 3). In more general axial defects, we will see that the basic features predicted by a cylindrical-defect profile are very similar. We also assume that the rays have only one reflection.

For a cylindrical axial extended defect like shown in Fig. 3, the overall surface profile may be described by:

$$
f(x)=\left\{\begin{array}{l}
y_{\mathrm{D}}+a_{\mathrm{D}} \sin \left(\arccos \frac{x_{\mathrm{D}}-x}{a_{0}}\right), x \in\left(x_{I}, x_{S}\right) \\
a_{0} \sin \left(\arccos \frac{x}{a_{0}}\right), x \in\left(-a_{0}, x_{I}\right) \cup\left(x_{S}, a_{0}\right)
\end{array},\right.
$$

where $a_{0}$ and $a_{\mathrm{D}}$ are, respectively, the radius of curvature of the wire and the defect, $\left(x_{\mathrm{D}}, y_{\mathrm{D}}\right)$ are the position of the centre of the defect, and $x_{I}, x_{S}$ are the defect limits on the $x$-axis. The way we will denote the size parameters of the defect in the figures are as follows: the width of the defect $w$, the height $t$ and the angular position $\gamma_{\mathrm{D}}$ (Fig. 3).

Some simulation results have been obtained following Eqs. (1)-(3). They are shown partially in Fig. 4 where we consider different defects in size $t$ but with the same wire and location. The bold line represents the sampling average of the lighter line (original simulation), calculated with a sampling band of $2^{\circ}$, representative of our experimental setup. In the Fig. 4 we increase the radius of the defect $a_{\mathrm{D}}$ keeping constant the wire radius $\left(a_{0}=200 \mu \mathrm{m}\right)$ and the defect width $(w=10 \mu \mathrm{m})$. This is equivalent to a decrease in the height (or depth) of the defect $t$. The height to width ratio of the defect determines the angular interval in which the scattered rays are distributed. Let denote it as $\Delta \psi_{t}$ (Fig. 4b). In this interval the scattered light interferes with the global reflection of the wire producing a speckle-like appearance on the intensity profile. More relevant for defect detection is the deep minimum in the intensity profile which corresponds geometrically to the location of the defect. In other words, with no defect, the light specularly reflected in that position would fill 

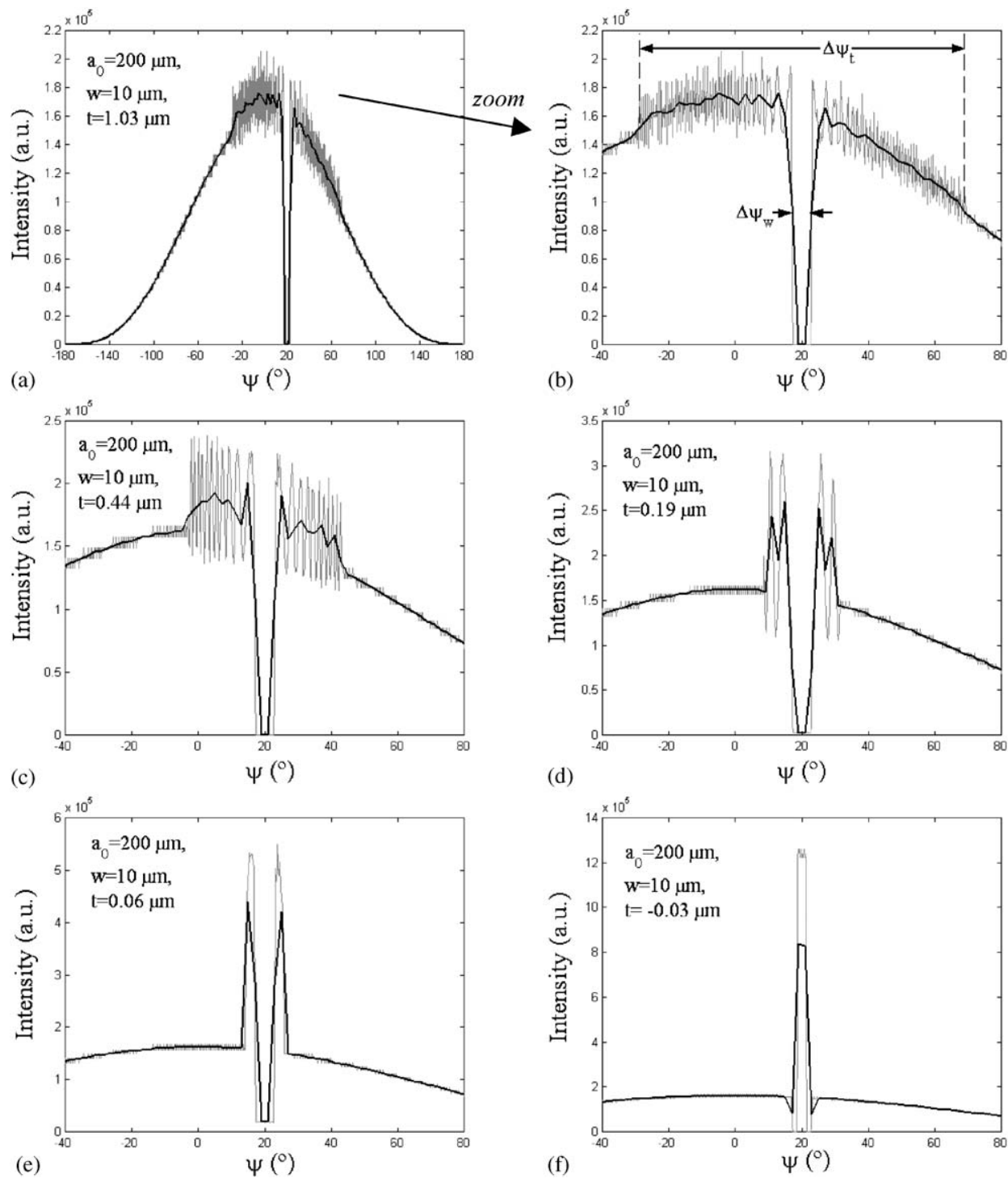

Fig. 4. Intensity profile calculated with Eqs. for several semi-cylindrical defects on a wire with $a_{0}=200 \mu \mathrm{m}, w=10 \mu \mathrm{m}, \gamma_{\mathrm{D}}=10^{\circ}$. The angular interval to compute the irradiance is $\Delta \psi=0.25^{\circ}$ (lighter line) and the sampling interval is $p=2^{\circ}$ (bold line). Total number of rays is $N_{0}=50000$. Defect parameters: (a) $t=1.03 \mu \mathrm{m}$, (b) detail of (a), (c) $t=0.44 \mu \mathrm{m}$, (d) $t=0.19 \mu \mathrm{m}$, (e) $t=0.06 \mu \mathrm{m}$, and (f) $t=0.03 \mu \mathrm{m}$.

the gap. The angular width of this dark fringe $\Delta \psi_{w}$ is twice the angular size of the defect, i.e. we have

$$
\Delta \psi_{w}=2 w / a_{0} .
$$


As the defect approach $t=0$ or equivalently $a_{0}=a_{\mathrm{D}}$ (no defect condition), the angular spread of the scattered light $\Delta \psi_{t}$ diminishes while, at the same time, it increases the interference modulation close to the defect gap Figs. 4(c)-(e). Therefore, high modulation peaks around a deep minimum are an indication of shallow defects, independently of its width. For $a_{\mathrm{D}} \gg a_{0}$ the defect is practically a flat surface reflecting the rays in a collimated way, what cause a maximum intensity peak in the specular defect position since $\Delta \psi_{t}<\Delta \psi_{w}$, surrounded by intensity minima (see Fig. 4f). We obtain the same intensity profiles for semi-cylindrical defects negatively curved (Fig. 5).

We see that the smallest defects which mean a little change in the slope distribution from the surface profile from a smooth surface, cause a big change around the specular no-defect situation on the corresponding intensity profile.

From a more generalized view we may explain the basic behavior as follows. For relatively big defects, the main feature at the reflected pattern is a gap in the intensity profile (which appears as a radial dark fringe in the projected cone), surrounded by an extended ripple- or speckle-like modulation due to the interference of the scattered light with the global smooth reflection. The minimum of intensity of this gap is always very close to zero. The extension of the ripple around such gap is proportional to the maximum slopes (positive and negative) of the defect profile, relative to the smooth expected profile of the wire. The defect slope distribution is related with the size parameters of the defect, i.e. we have bigger slopes for a big height to width ratio $(t / w)$.

For progressively shallower defects we encounter an increase in the intensity of the ripple modulation but restricted to closer surroundings of the defect intensity gap. At the same time, the minimum in the intensity profile is filled up and it may even transform to a maximum surrounded by minimums in the case of a defect consisting of a flattened strip (Figs. 4(d)-(f)).

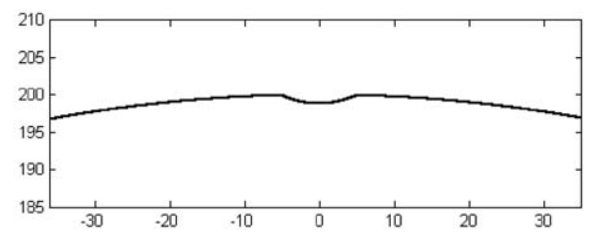

(a)

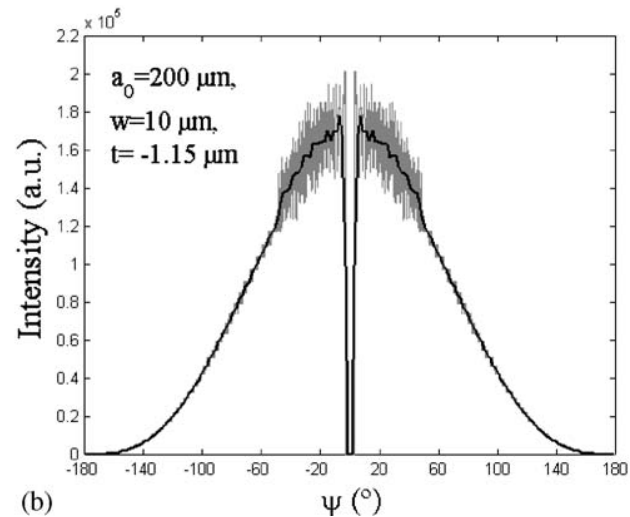

(b)

Fig. 5. (a) Surface profile for a defect on a wire like in Fig. $7\left(a_{0}=200 \mu \mathrm{m}, w=10 \mu \mathrm{m}, \gamma_{\mathrm{D}}=0^{\circ}\right)$ with $t=-1.15 \mu \mathrm{m}$. (b) Corresponding intensity profile for $\Delta \psi=0.25^{\circ}, p=2^{\circ}, N_{0}=50000$. 
In conclusion, defects with a big slope to width ratio $(t / w)$ produce a nearly uniform speckle-like spread $\Delta \psi_{t}$ quite extended. For $t / w$ small the scattering spread $\Delta \psi_{t}$ decreases and the interference modulation increases.

Some basic limitation to the resolution of the inspection technique based on this model may be seen in terms of the collimation of the incident beam, which should be below $\Delta \psi_{w}$. Other aspect of the presented results is that they are geometrically scalable. This means that from a geometrical point of view, a constant wire-defect angular relation will produce the same intensity pattern, i.e. when $w / a_{0}$ and $t / w$ are kept constants. For more general defect profiles, the basic features in the intensity pattern are expected to be described within the simple model developed for cylindrical defects.

\section{Experimental setup}

In the Figs. 6 and 7 we see the details of the industrial prototype we have developed. To obtain a whole surface inspection around the wire, we need in principle, two conical reflected beams coming from the reflection on opposite sides of the wire, because each reflection cone contain the information from the illuminated zone (i.e. $180^{\circ}$ around the wire). Nevertheless, at grazing incidence and also due to the diffraction effects which affect the rays close to the edges, we loss the information in these regions, so finally we need at least three incident beams (angularly equidistant at $120^{\circ}$ ) to obtain a view of the wire surface with the information complete.

A principal laser beam is split in three beams by means of a diffraction grating and these auxiliary beams are directed to the wire at slightly different oblique incidence angles while keeping a perimeter separation of $120^{\circ}$ by means of three adjustable

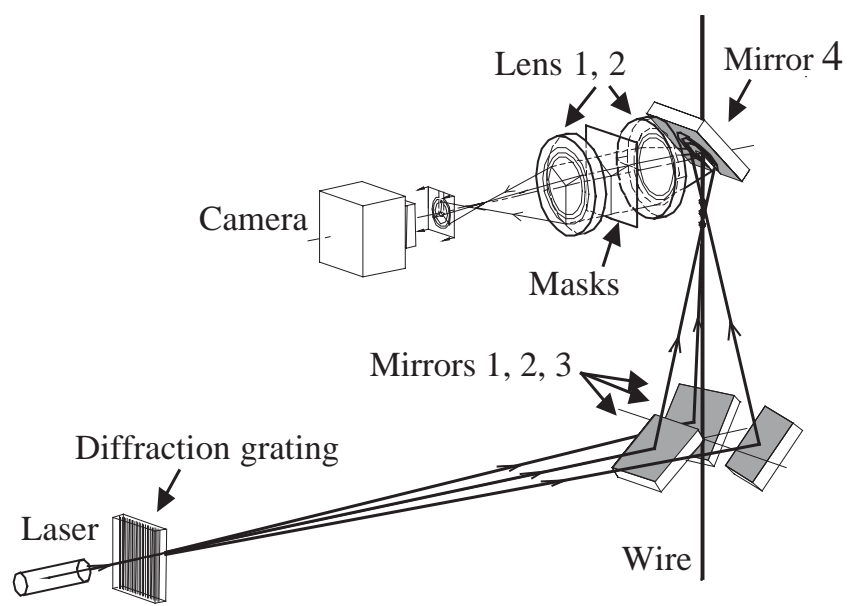

Fig. 6. Optical layout of the prototype and ray tracing for the triple conical reflection. 

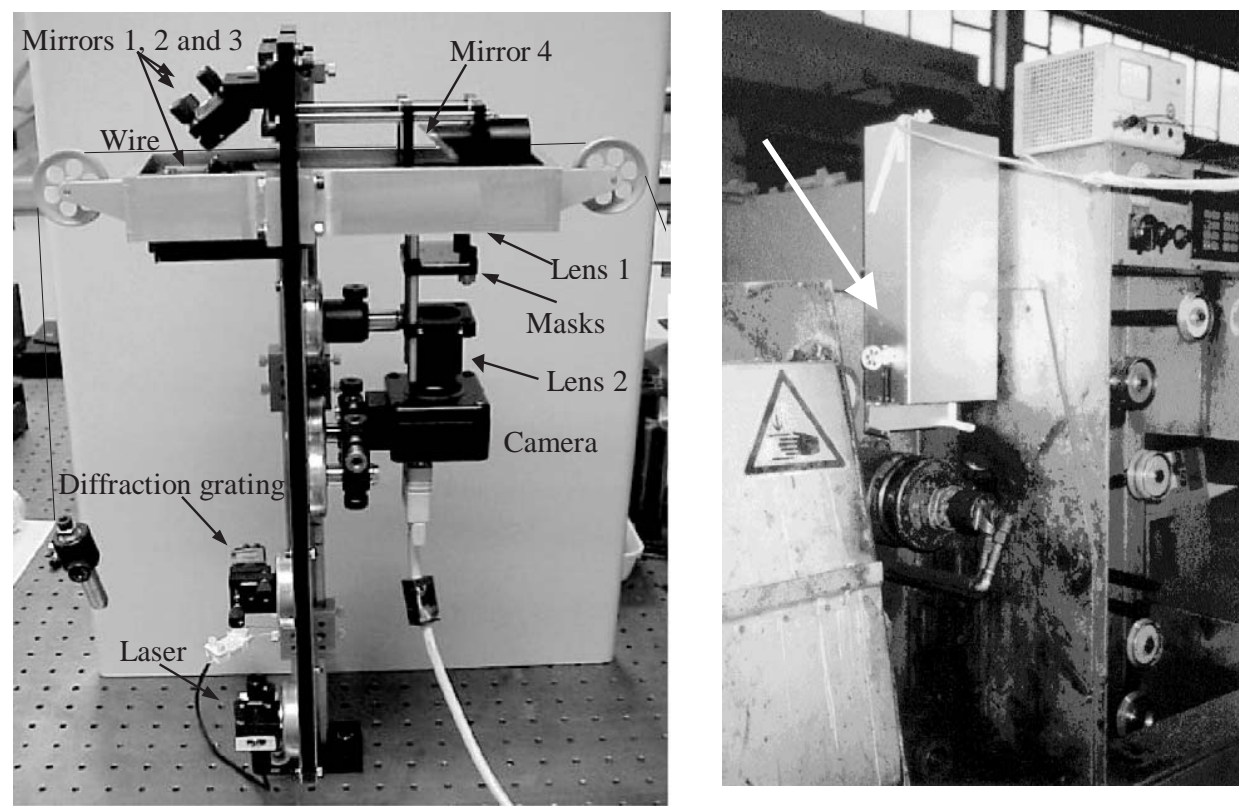

Fig. 7. Left: look at the prototype inside. Right: implementation of the prototype (covered) in a wire drawing machine.

mirrors (mirrors 1, 2 and 3 at Fig. 6). These beams have also small different incidence angles so that the obtained reflection cones are concentric to the wire but do not overlap.

Mirror 4 located on the wire axis deviates the reflection cones to an optical system of two lenses (lenses 1 and 2) which project the tree reflection cones on a CCD. The diffraction effects (impact point of the direct incident beam) are removed by masks located between the lenses 1 and 2 . A remarkable quality of the prototype is that it can be implemented directly in the production line of the wire without spinning it through the prototype, what facilitates its integration in the industry. This property is achieved by means of a breach aperture at mirror 4 that allows the wire to pass.

\section{Experimental results}

\subsection{Static operation}

Consider first one incident beam alone and a still wire. For a smooth wire, we obtain the reflected pattern shown in Fig. 8 and a corresponding smooth intensity profile. In Fig. 9 we find two main surface defects on a wire which give rise to the corresponding fluctuations of the intensity profile: a defect with big $t$ (absolute value) 

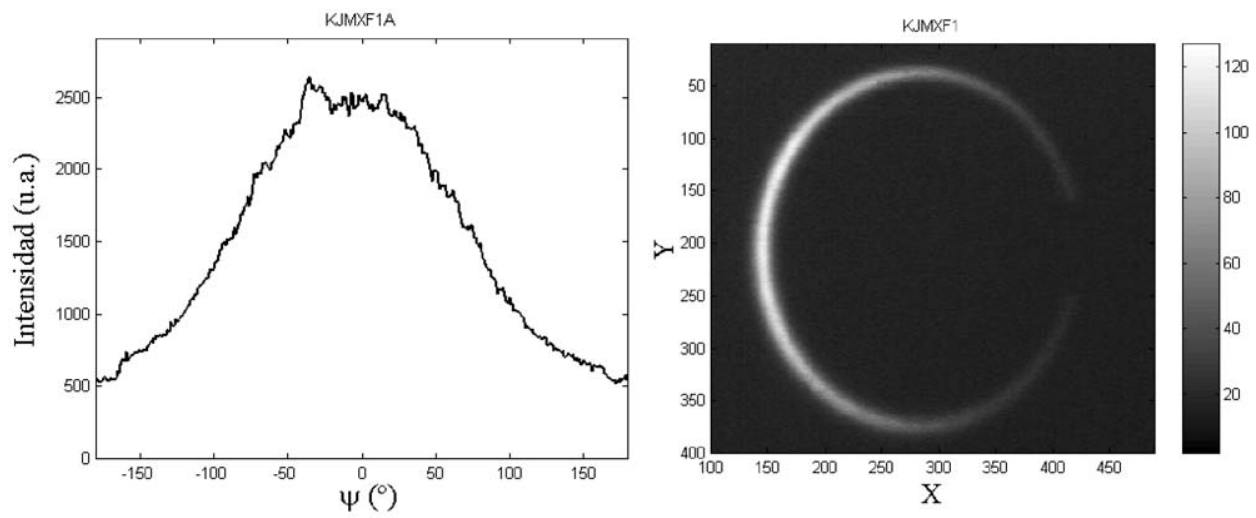

Fig. 8. Intensity profile (left) taken from the intensity pattern captured with a CCD camera (right) which intersect the conical reflected beam obtained experimentally from a smooth wire surface whose diameter is $400 \mu \mathrm{m}$.
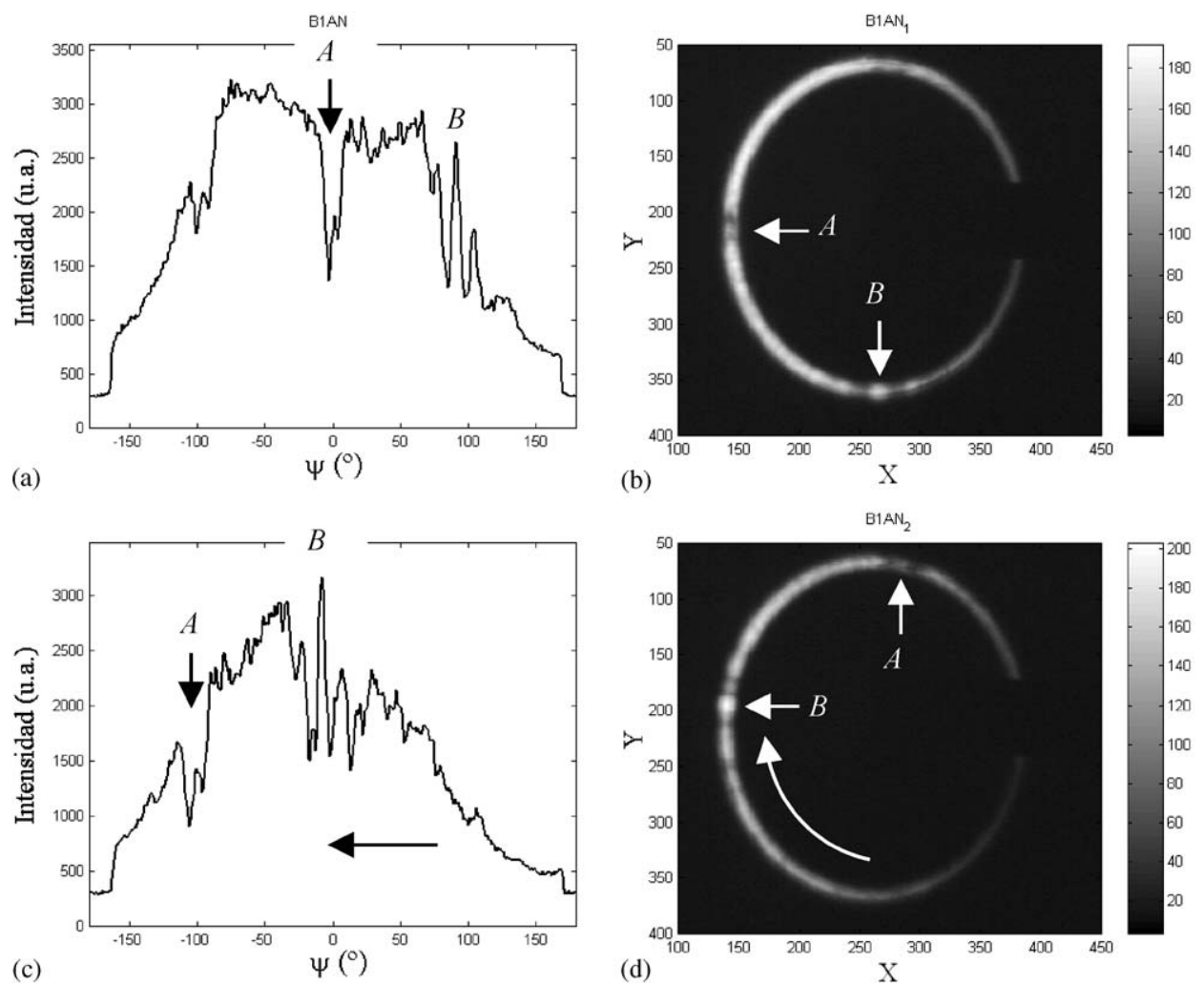

Fig. 9. Intensity profiles from a stainless-steel wire $\left(a_{0}=340 \mu \mathrm{m}\right)$ with axial extended surface defects. (a) Intensity profile of the image, (b) of the intersected reflection cone, (c) and (d) represents the same situation with the wire rotated $45^{\circ}$ around its axis (the reflected cone rotate then $90^{\circ}$ ). 
originates an intensity minimum $(\operatorname{defect} A$ ), while a defect with small $t$, probably a flat area, gives rise to an intensity peak between two minima (defect $B$ ) like we have predicted in Fig. 4. In Figs. 9(c)-(d) we rotate $45^{\circ}$ the wire around its axis causing the corresponding displacement of the defects $A$ and $B$ on the intensity profile in $90^{\circ}$. Therefore, we can appreciate a good correlation between the rotation of the reflected profile and the rotation of the wire confirming the basic geometric nature of the detection.

Fig. 10 shows the intensity profile and the image of the intersected conical reflected beam from a wire with a defect (indicated with an arrow) like that shown in Fig. 4d, where we can appreciate a minimum surrounded by intensity peaks. In this case, we may estimate the width $w$ of the defect $A$ from Eq. (4) and obtain $13 \mu \mathrm{m}$. Measurement of its width with an optical microscope give us $w=15 \pm 2 \mu \mathrm{m}$.

Typical images obtained with the three laser beams turned on are given in Fig. 11. In this figure we can see the three reflection cones and some "dark regions" (in
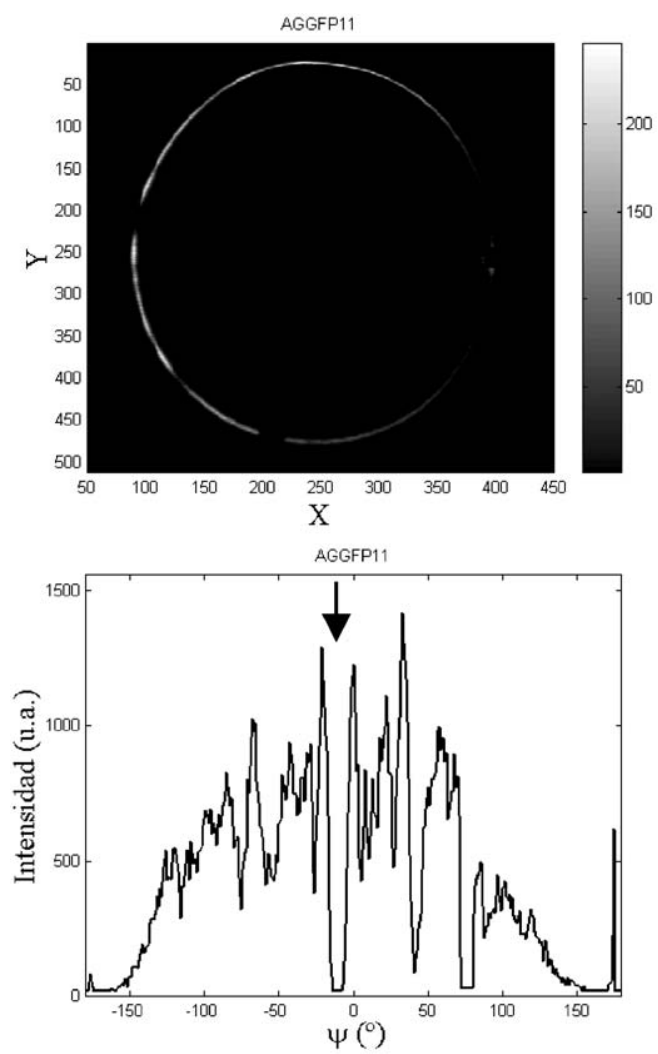

Fig. 10. Intensity profile of the image of conical reflection taken at a wire of $150 \mu \mathrm{m}$. Clear defects are differentiated. The arrow indicates a defect of $w=13 \mu \mathrm{m}$ (see text). 

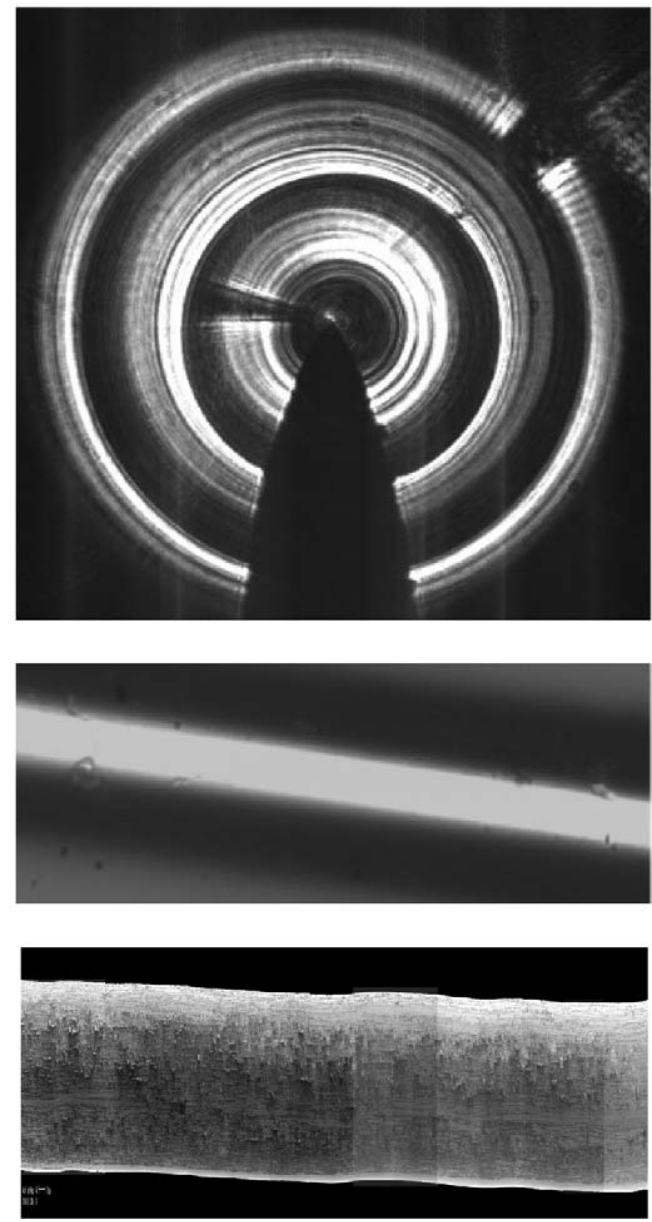

Fig. 11. Above: triple conical reflection image obtained for a good wire (see image at center). The wavy nature (below) or variable diameter of the wire produces the stratified appearance (onion-like) of the reflection pattern. The arrows indicate local point-like defects.

external ring: at 1 a o'clock, in internal ring: at 9 a o'clock, and in all the rings: at 6 a o'clock) which correspond to the masks and the mirror slit (mirror 4) and not to axial defects. We can appreciate that a smooth surface (Fig. 11 center) gives rise to a smooth angular intensity distribution, while a wire with a faulty surface (Fig. 12) gives a strong modulation of the annular intensity profile with several dark fringes or intensity gaps. Observe also that the waviness of a wire or diameter variation along its axis Fig. 11 (bottom) produces ring profiles with onion-like structure, although the wire surface in this case is very smooth. Finally, it is also possible correlate pointlike defects with point-like intensity gaps in the reflection cone (indicated by arrows in Fig. 11). 

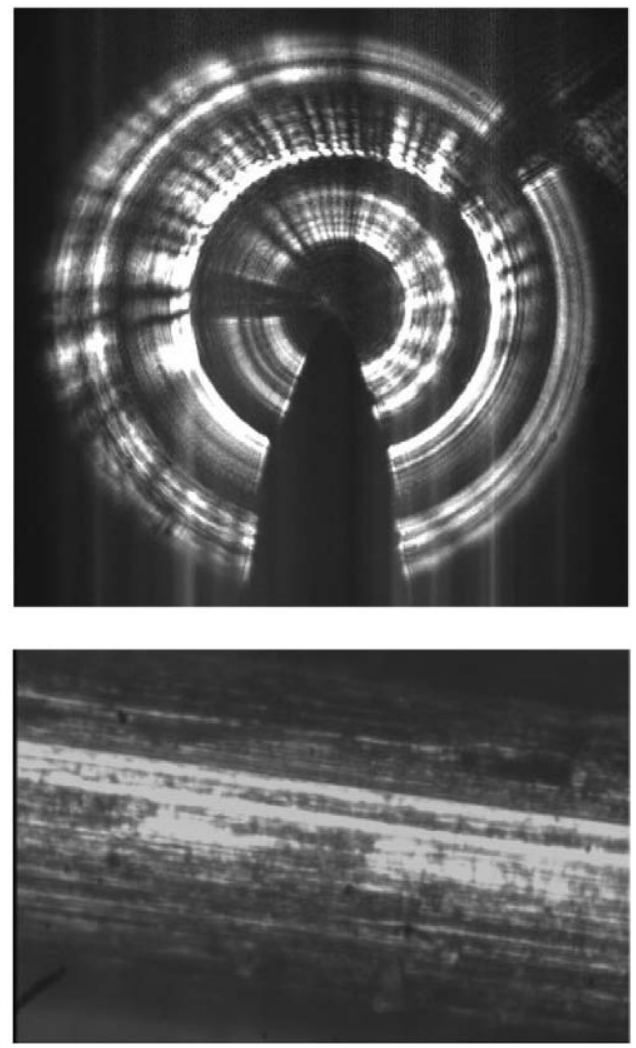

Fig. 12. Image obtained for a faulty wire corresponding to the wire surface shown below obtained with an optical microscope.

\subsection{Dynamic operation}

In practice, when the wire is in movement with a certain wire guiding speed $v$ trough the prototype, we have to consider several instabilities and smoothing effects. In the industrial tests performed we find out that it is possible to capture the three reflection cones sufficiently stabilized on the CCD by maintaining the wire sufficiently stretched. Under this situation the pattern generated by an axial defect is constant in dynamic operation (i.e. the smoothing affects to the drawing direction only).

We show some images captured by the prototype in production line. In Fig. 13 we show the image captured from a $30 \mu \mathrm{m}$ stainless-steel wire, increasing the wire speed from $v=2$ to $20 \mathrm{~m} / \mathrm{s}$, that lead to a smoothing of the intensity profile in radial direction at the CCD and fixed exposure time.

Finally, we scratch the surface of a wire ( $400 \mu \mathrm{m}$ stainless-steel) while drawing with a sand paper before entering the prototype. The effect can be appreciated in Fig. 14, before scratching (above) and while scratching (below). A significant variation in the 

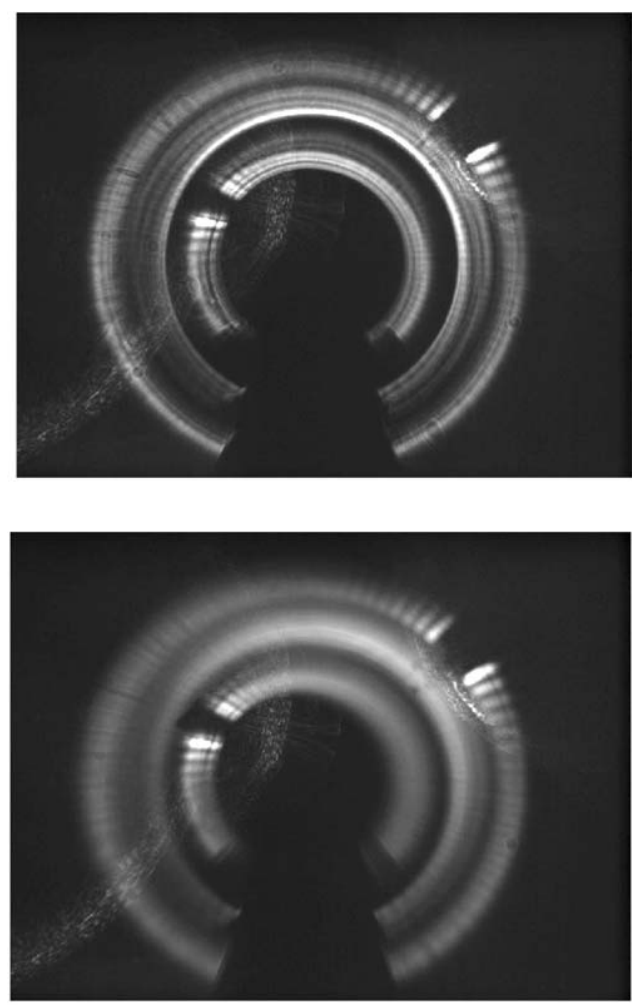

Fig. 13. Images captured by the prototype for different wire guiding speeds. Wire diameter is $30 \mu \mathrm{m}$. Above: wire speed is $v=2 \mathrm{~m} / \mathrm{s}$, the exposure time $t_{\exp }=10 \mathrm{~ms}$ corresponding to an axial length of $e_{\text {exp }}=20 \mathrm{~mm}$. Below: $v=20 \mathrm{~m} / \mathrm{s}, t_{\exp }=10 \mathrm{~ms}, e_{\exp }=200 \mathrm{~mm}$.

quality is observed in the reflection profiles. The scratching produces an increase of intensity gaps (Fig. 14).

\section{Conclusion}

We presented an integral development for the detection and quantification of axial defects on cylindrical metallic wires, in static or dynamic operation. We presented: (1) the development of a simple theoretical model although adequate for the interpretation of the reflection intensity patterns, (2) the development and implementation of an industrial prototype adapted for static and dynamic inspection and (3) some experimental results which corroborate the viability of the technique. The proposed model is valid within the geometrical approximation, i.e. for big enough defects and wires $\left(w>\sim \lambda(\sim 1 \mu \mathrm{m})\right.$ and $\left.a_{0}>\sim 3 \lambda\right)$.

The typical feature produced by an axially extended defect is the apparition of an intensity gap in the reflection profile which a width proportional to the width of the 

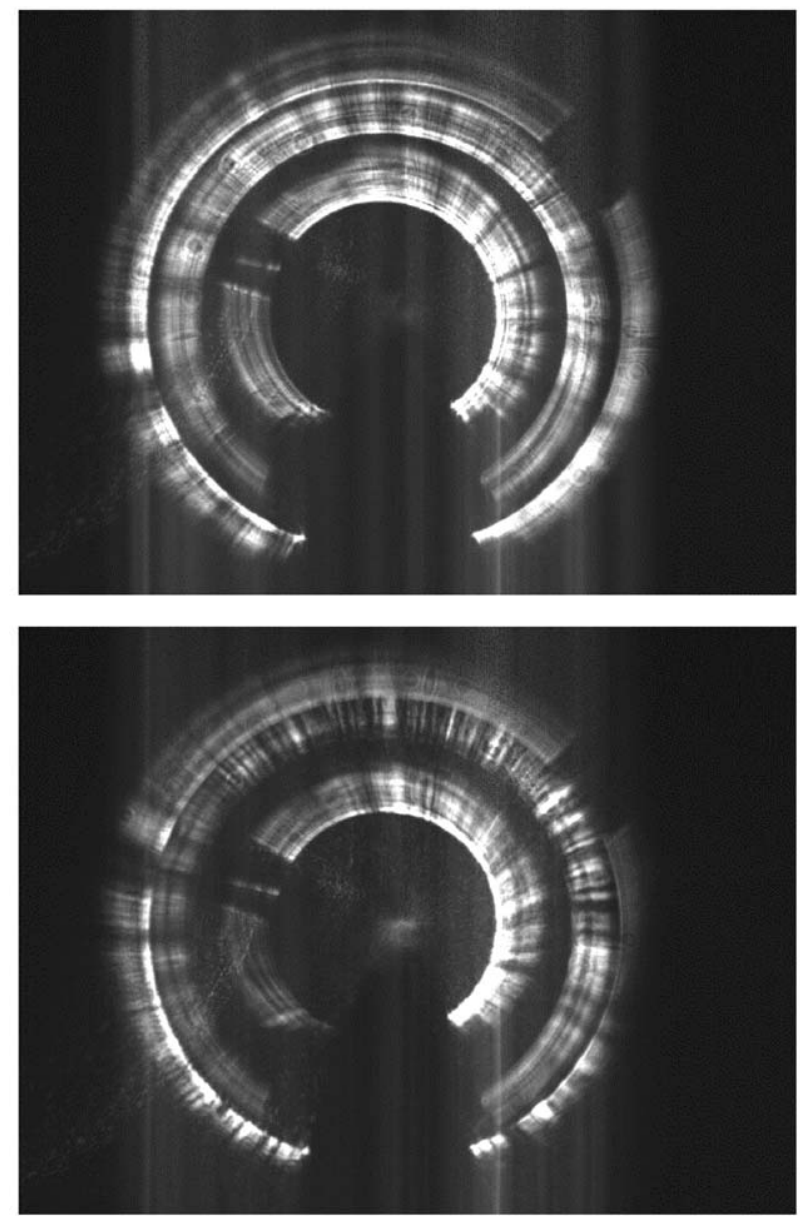

Fig. 14. Image captured by the prototype in a wire drawing machine before (above) and while (below) scratching it with a sandpaper. Wire diameter $30 \mu \mathrm{m}, v=1 \mathrm{~m} / \mathrm{s}, t_{\exp }=10 \mathrm{~ms}\left(e_{\exp }=10 \mathrm{~mm}\right)$.

defect and secondly, the apparition of a speckle or an interference-modulated pattern surrounding the intensity gap which informs of the relative height or depth of the defect.

\section{Acknowledgements}

The authors are grateful to the European Commission (EU) for the financial support of this work under the project SMT4-CT97-2184 (DEFCYL: Detection of Surface DEFects on CYLindrical Surfaces). The authors want to express their gratitude to Zumbach GmbH, Sprint Metal GmbH and Joh Pengg AG for their 
participation in the project and the cooperation in the industrial testing of the developed prototype.

\section{References}

[1] Bernabeu E, Sanchez-Brea LM, Siegmann P, Martinez-Antón JC, Gomez Pedrero JA, Wilkening G, Koenders L, Müller F, Hildebrand M, Herman H. Classification of surface defects on fine metallic wires. Appl Sur Sci 2001;180:191-9.

[2] Bernabeu E, Sanchez-Brea LM, Siegmann P, Martinez-Antón JC, Gomez Pedrero JA, Wilkening G, Koenders L, Müller F, Hildebrand M, Herman H. Surface Structures on fine and ultrafine wires, catalog printed by Editorial Complutense on the behalf of the Commission of the European Community. 2002;1, ISBN: 84-7491-679-8.

[3] Sanchez-Brea LM, Siegmann P, Rebollo MA, Bernabeu E. An optical technique for the detection of surface defects on thin metallic wires. Appl Opt 1999;39(4):539-45.

[4] Martinez-Anton JC, Siegmann P, Sanchez-Brea LM, Bernabeu E, Gómez-Pedrero JA, Canabal H. Inline detection and evaluation of surface defects on thin metallic wires. In: Höfling R, Jüptner WPO, Kujawinska M, editors. Optical measurement systems for industrial inspection II: application in production engineering, Proceedings of the SPIE (The International Society for Optical Engineering), vol. 4399. 2001. p. 27-34, Munich, Germany, ISBN: 0-8194-4094-9.

[5] Berlasso R, Perez-Quintián F, Rebollo MA, Raffo CA, Gaggioli NG. Study of speckle size of light scattered from cylindrical rough surfaces. Appl Opt 2000;39(31):5811-9.

[6] P. Siegmann. PhD dissertation, Universidad Complutense Madrid, Madrid, Spain, July 2002. 